\title{
Chapter
}

\section{NANOPARTICLE-MEDIATED SIRNA DELIVERY FOR LUNG CANCER TREATMENT}

Anish Babu1,2, Narsireddy Amreddy,2, Ranganayaki Muralidharan 1,2, Anupama Munshi' ${ }^{2,3}$, and Rajagopal Ramesh 1,2,4*

${ }^{1}$ Department of Pathology

2Stephenson Cancer Center, University of Oklahoma Health Sciences Center, Oklahoma City, Oklahoma 73104, USA

${ }^{3}$ Department of Radiation Oncology, University of Oklahoma Health Sciences Center, Oklahoma City, Oklahoma 73104, USA

${ }^{4}$ Graduate Program in Biomedical Sciences, University of Oklahoma Health Sciences Center, Oklahoma City, Oklahoma 73104, USA 


\section{Contents}

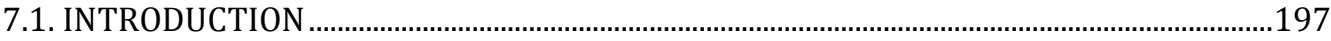

7.2. LIPOSOMES AS siRNA NANOCARRIERS...................................................................................198

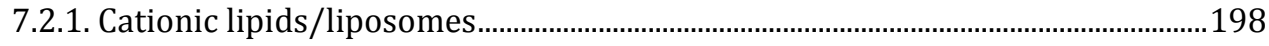

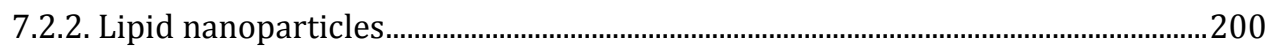

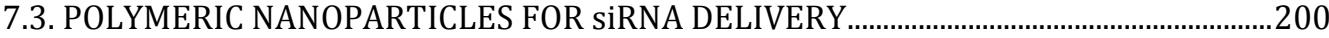

7.3.1. Poly(ethyleneimine)-based nanodelivery systems..................................................201

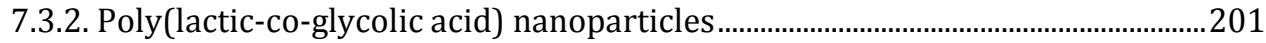

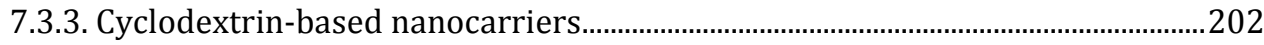

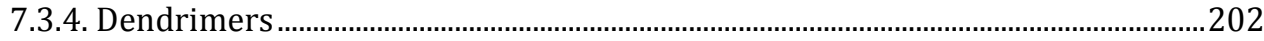

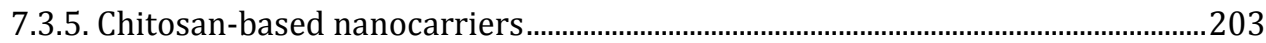

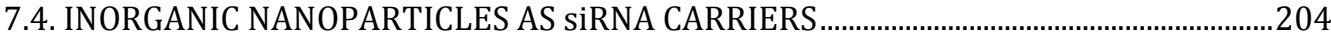

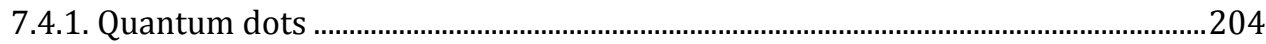

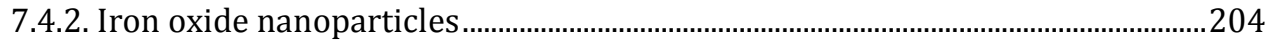

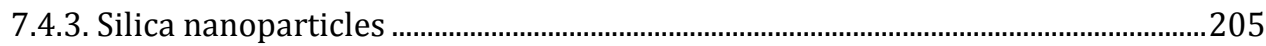

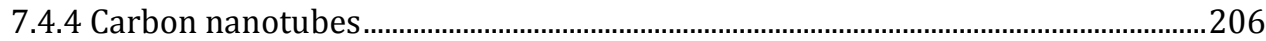

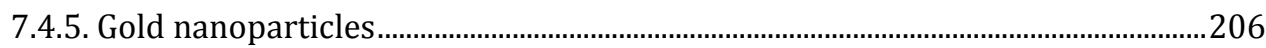

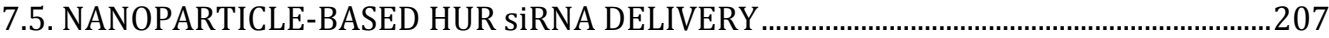

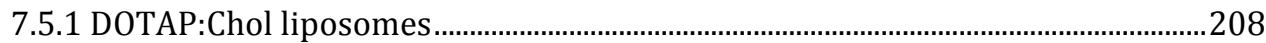

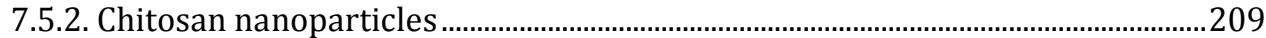

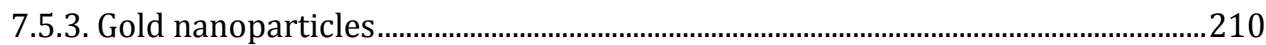

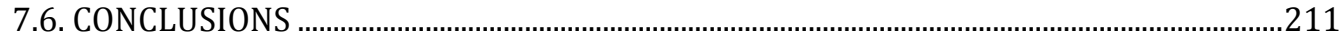

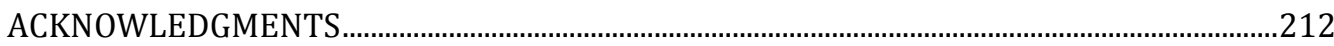

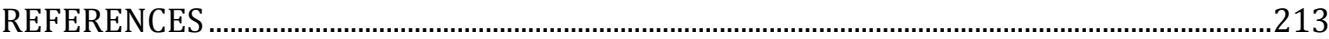




\subsection{INTRODUCTION}

RNA interference (RNAi) is a process by which the function of a specific messenger RNA (mRNA) is blocked by introducing a short fragment (21-23 nucleotides) of RNA with complimentary sequences into the cell cytoplasm. The cellular machinery involving the RNA-induced silencing complex (RISC) then processes this short RNA (shRNA) into antisense RNA fragments, which ultimately bind to the complimentary mRNA and prevent the translation of mRNA into specific proteins [1]. Small interfering RNA (siRNA) is a doublestranded RNA helix that has been heavily investigated as an RNAi tool for anticancer gene therapy [2]. siRNAs are designed to specifically target genes, causing effective downregulation of proteins that are involved in cancer pathology and progression. In the past decade, tremendous achievements have been made in the development of siRNA therapeutics for cancer therapy, as demonstrated by a number of human clinical trials in progress [3]. Successful application of RNAi depends on the effective intracellular delivery of siRNA, bypassing a number of biological barriers. In the laboratory, siRNA is more stable than native mRNA; however, siRNAs are prone to degradation by nucleases and have a short half-life of less than $1 \mathrm{~h}$ in the presence of plasma proteins. The macromolecular size, hydrophilicity, and negative charge of siRNA prevent effective transportation of naked siRNA across the cellular membrane. Therefore, an effective delivery vector is required for siRNA to be transported across physiological barriers to the desired cell cytoplasm. Viral vectors were the first siRNA delivery systems investigated thoroughly for their efficiency in targeted gene knockdown. Despite their high transfection efficiency, viral vectors were identified as potential elicitors of immune reactions in humans. Other safety concerns, such as the possibility of viral mutations, recombination, and oncogenic effects, also limited the use of viral vectors for siRNA therapeutic delivery [4]. Recent advancements in nanotechnology have driven the revolution in developing nanoparticle carriers for highly challenging siRNA delivery [5]. Current siRNA delivery systems can be categorized into two general classes: organic and inorganic nanoparticles. Commonly used siRNA nanocarriers, such as liposomes and polymer nanocarriers, are included in the class of organic nanoparticles, whereas metalbased nanoparticles, Quantum dots (QDots), carbon nanotubes, and mesoporus silica nanoparticles are inorganic nanoparticles. In this chapter, we will provide an overview of the current strategies of siRNA delivery using nanoparticle formulations and discuss our efforts in formulating nanoparticle-based siRNA delivery systems toward a recently recognized molecular target of cancer, the Human antigen R (HuR). 


\subsection{LIPOSOMES AS SIRNA NANOCARRIERS}

Liposomes or lipid nanoparticles are commonly used for siRNA delivery to mammalian cells. Liposomes are unilamellar or multilamellar micro-vehicles consisting of a phospholipid bilayer. Liposomal nanocarriers have been extensively used to enhance efficient drug delivery since they are biocompatible [6]. These amphiphilic phospholipids have a hydrophobic tail and a hydrophilic polar head. They form a bilayer in water, with the hydrophobic tails facing each other and the hydrophilic side facing towards water. Because of their shape, size, and surface characteristics, these liposomes have the ability to deliver different payloads, including chemotherapeutic drug, DNA, siRNA, shRNA, and proteins. Liposomal carriers offer several advantages. Liposomes prevent enzymatic degradation of siRNA, support high siRNA loading, allow preferential accumulation of siRNA at the tumor site, promote endosomal escape resulting in efficient cytoplasmic delivery, and provide a safe and effective systemic delivery [7]. Molecules containing several amines per head group, with slight spacing between the amine groups, can bind to the negatively charged backbone of siRNA more efficiently than the lipids containing a single positive charge per head group. The stability of the positively charged lipids may be enhanced by the addition of the neutral lipid (helper lipid) to reduce the repulsion between similar charges in the lipid bilayer. The addition of cholesterol, which takes up residence in the hydrophobic region in the bilayer, improves the stability of the carrier and facilitates the cellular uptake of siRNA.

\subsubsection{Cationic lipids/liposomes}

Cationic liposomes are non-viral delivery systems that are extensively used to deliver RNAi [7]. Cationic lipids can self-assemble with negatively charged DNA and siRNA to form lipoplexes by electrostatic interaction, and enhance transfection efficiency. Almost two decades ago, Malone and colleagues demonstrated the use of cationic lipids in nucleic acid delivery towards mammalian cells [8]. Later, the same group described the process of lipofection in detail, using cationic lipid $N$-[1-(2,3-dioleyloxy)propyl]- $N, N, N$ -trimethylammonium chloride (DOTMA) to deliver DNA and RNA into mouse, rat, and human cancer cells [9]. Since then, liposomes have been used as gene delivery vehicles for many biomedical applications. Optimizing the lipid composition, size, charge, payload-to-lipid ratio, and the targeting moiety will provide an efficient liposomal system for siRNA delivery. The most successful commercial transfection agent is a liposomal formulation, Lipofectamine 2000, or its advanced version, RNAiMAX [10]. siRNA can rapidly complex with Lipofectamine 2000 to form lipoplexes because of the strong anionic-cationic interaction. While possessing a strong positive charge for efficient complexation with siRNA and promoting an enhanced gene silencing effect, cationic lipids/liposomes are rapidly cleared from the circulation and are toxic to cells [11]. Anionic lipids or neutral lipids show better stability in the 
circulation; however, the weak interaction between nucleic acidanionic/neutral lipids may result in the premature release of nucleic acids into the circulation. Thus, cationic lipids are still the leading choice for siRNA delivery formulations.

Studies from our lab have shown that the cationic lipid carrier $N-[1-(2,3-$ -dioleoyloxy)propyl]- $N, N, N$-trimethylammonium chloride: Cholesterol (DOTAP:Chol) can deliver the tumor suppressor genes p53, FUS1, and $M D A 7 / I L-24$ to lung tumor models [12-14]. Our studies demonstrated that DOTAP:Chol nanocarriers were selectively taken up by lung tumors without causing toxicity to the surrounding lung tissues, and resulted in increased transgene expression. In a typical study, the specificity of DOTAP:Chol liposomes was heightened by modification with the targeting moiety anisamide, enhancing the cellular uptake in lung cancer cells overexpressing sigma receptors [15]. A Phase I clinical trial was conducted using DOTAP:Chol-TUSC2 tumor suppressor gene complexes for treating human lung cancer [16]. The DOTAP-Chol-TUSC2 treatment resulted in transgene and gene product expression, specific alterations in TUSC2-regulated pathways, and antitumor effects. Besides our studies, numerous cationic liposomal systems have been investigated for gene delivery applications in cancer in vitro and in vivo. Some excellent reviews summarized the recent advances in cationic lipids/liposomes in gene delivery [17-19].

The efficiency of cationic liposomes in gene delivery can be further improved by the addition of poly(amino acid)-conjugated poly(ethylene glycol) (PEG) chains. For example, poly(L-arginine)-conjugated PEG-DOTAP/DOPE:Chol liposomes demonstrated enhanced intracellular uptake and low cytotoxicity compared with unmodified cationic liposomes in cancer cells [20]. PEG modification of liposomes has many advantages, such as preventing aggregation, enhancing shelf life, prolonged blood circulation time, reduced opsonization, slow clearance, and acting as a linker for the further modification of liposomes. Targeting ligands can be attached to the extremity of the PEG chains and interact with antigens or receptors overexpressed on the surface of the cancer cells. Incorporation of excess PEG-phospholipids will disrupt the integrity of the lipid membrane, due to its detergent-like properties, which will increase premature drug release and membrane permeability [21]. However, the degree of surface PEGylation is usually less than $5 \mathrm{~mol} \%$, in order to preserve the liposomes' integrity [22]. Various formulations of sterically stabilized PEG liposomes have been used for the systemic delivery of nucleic acid for gene silencing [23]. Atu027 is a DSPE-PEG-chains-stabilized, lipoplexed siRNA targeting protein kinase3 (PKN3). Atu027 underwent Phase I clinical trials in patients with cancer and was demonstrated to have a safe clinical profile, which might be partly attributable to the presence of the DSPE-PEG component [24]. This silencing therapeutic is currently undergoing Phase 2 trials as a treatment for advanced solid and metastatic cancers [25]. 


\subsubsection{Lipid nanoparticles}

Lipid nanoparticles have been proposed as alternative siRNA carriers [19]. Structurally, lipid nanoparticles are slightly different from liposomes, as lipid nanoparticles are composed of emulsified solid-lipid particles in aqueous dispersion. Lipid nanoparticles effectively protect the incorporated nucleic acids from nuclease attack and are known to modulate its releasing properties [26]. They are usually prepared from physiologically well-tolerated lipids, such as triglycerides like Tristearin, Compritol 888 ATO, and Dynasan 112, carnauba wax, cetyl alcohol, cholesterol, and cholesterol butyrate. Recently, solid tristearin lipid nanoparticles have been tested for sustained release of siRNA in vivo [26]. The siRNA was loaded into tristearin nanoparticles using an ion pairing approach, with the help of the cationic lipid DOTAP. The tristearin-DOTAP nanoparticle demonstrated extended release (10-13 days) of siRNA in a mouse model. Another study investigated the efficacy of a lipid nanoparticle-siRNA formulation in silencing androgen receptor (AR) protein in human prostate cancer cell lines and xenograft models [27]. Researchers screened a panel of cationic lipids and found that lipid nanoparticles that contain ionizable cationic lipid 2,2-dilinoleyl-4-(2-dimethylaminoethyl)-[1,3]-dioxolane (DLin-KC2-DMA) showed the highest gene silencing efficiency in vitro. In the next step, they demonstrated its transfection efficiency in xenograft tumors, producing good gene silencing efficiency. This study claimed to be the first in vivo use of a lipid nanoparticle delivery system for silencing AR gene expression. siRNA-incorporated lipid nanoparticles have also been used to achieve synergistic therapeutic effects in anti-cancer combination therapy with co-loaded drug. A typical strategy used QDots incorporating siRNA/drug-loaded lipid nanoparticles for combinatorial therapy of human lung cancer cells [28]. The nanoparticles were able to co-deliver paclitaxel and BCl2 siRNA, and exhibited synergistic anticancer effects. In addition, the fluorescence exhibited by the QDots was helpful in intracellular localization of lipid nanoparticles in cancer cells. Such an application of lipid nanoparticles holds great promise in the field of cancer theranostics.

\subsection{POLYMERIC NANOPARTICLES FOR SIRNA DELIVERY}

Polymeric nanoparticles have been widely investigated for siRNA delivery systems. siRNA is either electrostatically bound with the positively charged functional groups of polymers, or encapsulated within the polymer matrix of nanoparticles. Some commonly used polymers include poly(ethyleneimine) (PEI), PLGA (poly(lactic-co-glycolic acid)), cyclodextrin polymers, branched dendritic polymers, and chitosan. The unique properties of each of these polymers allowed researchers to investigate even the combined benefits of two or three polymers for efficacious siRNA delivery. 


\subsubsection{Poly(ethyleneimine)-based nanodelivery systems}

The most studied polymer for siRNA delivery systems is PEI. PEI is a synthetic polymer with the ability to electrostatically interact with negatively charged siRNA due to its cationic ability to form nanoscale complexes. Both linear and branched PEIs have been used as siRNA nanocarriers. These PEIs demonstrate a high buffering capacity to bypass the endosomal barrier. However, the clinical application of PEI is limited, because its membrane-permeabilizing nature is toxic for cells [29]. Tremendous efforts have been devoted to modifications of PEI without affecting its nucleic acid condensing ability, in order to reduce toxicity and to achieve biodegradability. Recently, the introduction of disulfide bonds (S-S) in PEI has been shown to increase its biodegradability and reduce its toxicity, rendering this polymer more promising for efficacious gene delivery [30]. Moreover, due to the siRNA condensation ability of PEI, it has been used as a major component of many gene delivery systems. Combination with other biocompatible polymer(s) not only reduced PEI's toxicity, but also improved gene delivery efficacy [31,32]. For example, a phospholipid dioleoyl-phosphatidylethanolamine (DOPE) conjugated with PEI has been investigated for P-glycoprotein gene-targeted siRNA delivery to reverse drug resistance in breast cancer cells. The transfection efficiency of the PEI carrier, which was otherwise ineffective against doxorubicin-resistant MCF-7 breast cancer cells, was enhanced upon conjugation with DOPE [31].

\subsubsection{Poly(lactic-co-glycolic acid) nanoparticles}

In contrast to PEI, PLGA is recognized as a safe polymer and is FDA-approved for human use. PLGA is one of the most highly investigated polymers for drug and gene delivery applications [33]. PLGA nanoparticles are known for their biodegradability and biocompatibility, and employ mild formulation techniques. A simple interfacial deposition or double emulsion technique can be followed to prepare siRNA-loaded PLGA nanoparticles. However, the poor siRNA loading efficiency is a matter of concern, mainly because PLGA cannot electrostatically interact with siRNA [34]. Moreover, PLGA nanoparticles show moderate efficiency in endosomal escape and cytoplasmic release of siRNA, which ultimately lead to low transfection efficiency. Nevertheless, researchers have shown that modification of PLGA nanoparticles with positively charged components enhance the efficiency of siRNA delivery. Poly(ethyleneimine), cationic lipids, and chitosan are important cationic agents that are used for PLGA nanoparticle modification. A recent study showed that coating PLGA nanoparticles with lipid improves siRNA encapsulation efficiency [35]. Another group investigated PLGA modification housing PEI for co-delivery of signal transducer and activator of transcription 3 (STAT3) siRNA and paclitaxel to drug-resistant lung cancer cells. This PLGA-PEI combination was effective in co-delivering STAT3 siRNA and paclitaxel, resulting in downregulation of STAT3 expression and controlled release of paclitaxel [36]. 


\subsubsection{Cyclodextrin-based nanocarriers}

Cyclodextrin, a natural polymer derived from cellulose by bacterial enzymatic digestion, is an attractive delivery system due to its structural characteristics, excellent biocompatibility, and water solubility. While the typical structure, which is truncated and cone-like with a cavity in the center, helps to form inclusion complexes with hydrophobic anticancer drugs, the amidine functional groups allow for the efficient condensation of nucleic acids. The large number of functional groups in cyclodextrin polymer allow for the bioconjugation of ligands or antibodies for targeted delivery. Cyclodextrin was approved for clinical trials examining siRNA delivery in cancer treatment, before any other cationic polymer [3]. The targeted cyclodextrin containing that polymer-siRNA delivery system, denoted as CALAA-01, is currently being investigated in clinical trials for cancer treatment [37]. CALAA-01 has four different components: cyclodextrin, siRNA, steric stabilization agent poly(ethyleneglycol) (PEG), and human transferrin (Tf) as a targeting ligand. These components self-assemble to form a nanocomplex. The CALAA-01 nanoparticle carries siRNA for targeted disruption of the M2 subunit of ribonucleotide reductase, designed to inhibit tumor growth. The preliminary study demonstrated that this cyclodextrin polymer-siRNA therapeutic had a better safety profile with low kidney toxicity in humans than was seen in preclinical observations in animals [37]. Apart from the cyclodextrin-polymer nanoparticles, Li et al. (2013) investigated the effect of PEI-conjugated cyclodextrin on siRNA delivery [38]. The study used FDA-approved 2 -hydroxypropyl- $\beta$-cyclodextrin (HP- $\beta$-CD) and low molecular weight PEI to synthesize an HP- $\beta$-CD /PEI siRNA nanocomplex for targeted cancer therapy. The nanoparticle demonstrated good in vivo stability and efficient gene knockdown, leading to tumor growth inhibition in a mouse model [38].

\subsubsection{Dendrimers}

Dendrimers form another class of polymer-based siRNA nanoparticle system of synthetic origin [39]. Typically, a dendrimer molecule consists of a central core from which repetitive branch units arise to a predetermined branch number, known as generations. The internal cavities thus formed can be utilized for encapsulation of small molecules or drugs, and the end functional groups of branchlets determine whether they can interact with nucleic acids. Thus, a dendrimer can be precisely designed for siRNA delivery by making them cationic for efficient condensation and protection of siRNA. Different kinds of dendrimers, including poly(amidoamine) (PAMAM), poly(L-lysine), poly(propyleneimine), carbosilane, triazine, and poly(glycerol)-based dendrimers, have been explored for siRNA delivery [40]. Among these, PAMAM is the best studied, since it is relatively easy to synthesize and is available as a fully characterized commercial product. However, the use of cationic dendrimers is limited due to toxicity, which greatly depends on their surface chemistry [41]. Surface modification with PEG has shown to be effective in 
reducing PAMAM dendrimer toxicity and improving gene silencing efficiency [42]. Another study demonstrated that arginine modification of low generation dendrimers resulted in effective gene delivery vectors both in vitro and in vivo [43]. Further, arginine modification also reduced the toxicity associated with PAMAM dendrimers, demonstrating their potential as a safe nanovector for siRNA delivery.

\subsubsection{Chitosan-based nanocarriers}

Chitosan, a natural polysaccharide polymer, has been extensively studied in the field of gene delivery research. The strong positive charge of chitosan allows its electrostatic interaction with siRNA to form self-assembled nanocomplexes. These nanocomplexes are able to protect siRNA from degradation and act as carriers. The important advantages of chitosan are its biocompatibility, biodegradability, and strong mucoadhesive nature. Chitosan has recently gained immense interest, as it is generally recognized as safe (GRAS) by the FDA and is a cost-effective delivery system for RNAi candidates. Another unique advantage of chitosan, apart from its ability to form nanocomplexes, is that it forms stable nanoparticles in the presence of strong crosslinkers or counter ions, such as tripolyphosphate and sodium sulfate. Crosslinked nanoparticles provide significant protection and enhanced loading of siRNA, possibly through the combined mechanisms of electrostatic interaction and entrapment in the entangled mass of chitosan-TPP [44]. Despite these advantages, limitations do exist, including insolubility in neutral and physiological $\mathrm{pH}$ and a slow endosomal escape rate [45]. However, chemical modifications and improvements in formulation have been developed to overcome the limitations of native chitosan in siRNA delivery applications. The solubility of chitosan siRNA carriers can be increased by PEG modification or by using chitosan derivatives, such as glycol-chitosan. However, siRNA loading was significantly impaired with such systems, as many of the available amine groups are utilized in the bioconjugation process, and the presence of free hydroxyl groups has hindered the electrostatic interaction between siRNA and chitosan. The endosomal escape mechanism is highly influenced by protonation of amines in cationic polymers by acidic conditions in endosomes. Thus, a low charge density of chitosan polymer allows only moderate efficiency in endosomal escape. Modifications of chitosan polymer with suitable polymers or molecules to bypass the endosomal barrier have resulted in enhanced siRNA delivery and transfection efficiency in cancer cells. Grafting of PEI with chitosan polymers was shown to be a successful strategy to increase the endosomal escape and transfection efficiency [46]. Similarly, conjugation of histidine molecules to chitosan polymer enhanced the gene transfection efficiency of the chitosan carrier, perhaps by aggravating the endosomal escape mechanism [45]. 


\subsection{INORGANIC NANOPARTICLES AS SIRNA CARRIERS}

In recent years, inorganic nanoparticles have emerged as potential siRNA delivery systems. QDots, iron oxide, gold, carbon nanotubes (CNTs), and mesoporus silica nanoparticle are all inorganic nanoparticles that are commonly used for gene/drug delivery applications. Inorganic nanoparticles can also be used in image-guided therapy. For example, gold, iron oxide, or QDot nanoparticles possess unique electronic, optical, and magnetic properties that enable real-time imaging of siRNA/drug delivery. The dual-purpose characteristics of inorganic particles, citing suitable examples, are discussed below.

\subsubsection{Quantum dots}

Semiconductor QDots, which are light-emitting nanoparticles, have been increasingly used as biological imaging and labeling probes due to their high quantum yield. Researchers recently discovered that QDots can also be used as efficient siRNA delivery material for tumor cells [47]. Thus, QDots act as a dual-purpose platform for the delivery and localization of siRNA inside tumors. This strategy allows for the monitoring of successful transfection in real time. In a typical study, QDots were decorated with siRNA and tumor-homing peptides were targeted to xenograft tumors in mice [48]. This study employed siRNA conjugation with the PEGylated QDot scaffold through a chemical cross linker, rather than using electrostatic interaction, as reported in many other schemes. Escaping the endosomal barrier is a challenge for QDot-delivered siRNA. In order to facilitate proper intracellular transport and endosomal escape of siRNA, QDots were coated with a proton-absorbing polymer in a different strategy [49]. It was noted that the creation of this proton-sponge coating enhanced the gene silencing efficiency of the QDot-siRNA complex 10-20 folds, with a simultaneous reduction in cytotoxicity, compared with commercial transfection reagents.

\subsubsection{Iron oxide nanoparticles}

Iron oxide nanoparticles were initially developed as feasible imaging agents due to their intrinsic magnetic properties. The superparamagnetic nature of iron oxide nanoparticles was explored for use with $\mathrm{T}_{2} \mathrm{MRI}$ imaging. Later, magnetic iron oxide nanoparticles demonstrated their potential in drug and gene delivery applications. The large surface area of these nanoparticles facilitates multiple functional modifications, such as enabling the conjugation of targeting molecules, drugs, siRNA, and DNA. Thus, the versatile nature of iron oxide nanoparticle delivery systems offers the potential for magnetically guided targeting coupled with gene therapy [50], drug delivery, MRI imaging, and magnetic hyperthermia. Boyer et al. (2010) synthesized iron oxide nanoparticles (IONPs) surface-coated with two different kinds of polymers [51]. The inner layer coating for IONP was poly(dimethylaminoethyl acrylate) 
(P(DMAEA)), and the outer layer was coated with poly(oligoethylene glycol) methyl ether acrylate (P(OEG-A)). The hybrid nanoparticles thus formed were used to create an IONP-siRNA nanoparticle complex. These IONP-siRNA nanocarriers demonstrated good transfection efficiency in a substrate adherent s(type) clone of SKNSH (SHEP) human neuroblastoma cells under an external magnetic field. After attaching polymers and siRNA to the IONPs, studies showed high proton transverse relaxation enhancement $\left(\mathrm{T}_{2}\right)\left(160 \mathrm{~s}^{-1}\right.$ per $\mathrm{mM}$ of $\mathrm{Fe}$ ) [51]. Apart from magnetic-guided targeting, iron oxide nanoparticles have also shown promise in the peptide-targeted delivery of siRNA. Lee et al. (2009) synthesized manganese-doped magnetic iron oxide (MnMEIO) nanoparticles, and then conjugated these with cancer-specific targeting moieties: the Arg-Gly-Asp (RGD) peptide, which targets $\alpha \mathrm{v} \beta 3$ receptors, and Cy5-dye-labeled siGFP, which inhibits the expression of green fluorescence protein (GFP) [52]. The nanoparticle formulation (MnMEIO-siGFP-Cy5/PEG-RGD) showed specific cell uptake and targeted gene silencing in MDA-MB-435 breast cancer cells expressing $\alpha \mathrm{v} \beta 3$ receptors [52].

\subsubsection{Silica nanoparticles}

Another promising material for siRNA delivery for cancer therapy is mesoporous silica. Mesoporous silica nanoparticles (MSNs) can be used for multiple therapies simultaneously, which allows for combinations of drug/DNA and drug/siRNA, since the silica nanoparticles have high drug-loading capacity $[53,54]$. The cationic functionalization of MSNs allows for the binding of nucleic acids on the particles' surface, and the porous structure permits the encapsulation of large amounts of drug molecules within the particles. In a feasibility study for co-delivery of nucleic acid(s) and drug, Bhattarai et al. 2010 developed a polycation and PEG-modified mesoporous silica nanoparticle [55]. The MSNs were complexed with plasmid DNA (luciferase) or siRNA (luciferase targeting) through electrostatic interaction, and were then loaded with lysosomotropic agent chloroquine (CQ) to treat B16F10 murine melanoma cells. The co-delivery of CQ and the siRNA significantly improved the transfection efficiency and silencing activity of the complexes compared with CQ-free MSNs. This study thus demonstrates an important strategy for combination (siRNA/DNA-Drug) cancer therapy using a single nanoparticle platform [55]. In the same year, another study reported the co-delivery of P-Glycoprotein (Pgp)-targeted siRNA and anti-cancer drug doxorubicin using a cationic MSN [53]. The cationic modification of MSN was performed using a PEI coating for efficient siRNA complexation and delivery. The effective delivery of Pgp-targeted siRNA along with controlled release of doxorubicin mediated by MSNs improved the chemosensitivity of the drug-resistant KB-V1 cancer cell line towards doxorubicin. A new strategy of packaging siRNA into MSN's mesopores has been recently developed by Li et al. (2011) [56]. The siRNA was packed under strong dehydrated solution 
conditions, followed by capping the siRNA-MSN using a cationic PEI polymer. The siRNA-MSN-PEI nanoparticle demonstrated good siRNA protection efficiency and efficient cytoplasmic delivery of siRNA. High-efficiency knockdown of enhanced green fluorescent protein (EGFP) gene and the $\mathrm{BCl} 2$ gene were achieved using the siRNA-MSN-PEI nanoparticle [56]. A further enhancement in transfection was obtained when they conjugated a fusogenic peptide, KALA, to the siRNA-MSN-PEI nanoparticle to treat lung cancer and human cervical cancer cell lines [57].

\subsubsection{Carbon nanotubes}

Carbon nanotubes (CNTs) are novel nanomaterials, with unique physical and chemical properties compared with other carbon materials. Recent research has demonstrated that, due to their nano-needle structure, CNTs easily cross the plasma membrane and translocate into the cytoplasm of target cells, using an endocytosis-independent mechanism without inducing cell death. Interestingly, several functionalized CNTs have already been generated and tested for siRNA delivery. Nucleic acids can be conjugated to phospholipid-functionalized carbon nanotubes via disulfide bonds, which are cleavable at the cell cytoplasm [58]. This strategy allows the single-walled carbon nanotube (SWCNT) to successfully deliver DNA oligonucleotides across the cell membrane and permits efficient nuclear translocation of DNA. Notably, DNA-phospholipid modified SWCNT demonstrated high RNAi efficiency compared with a commercial transfection agent, Lipofectamine ${ }^{\circledR}$. The same strategy of siRNA conjugation and delivery was again found successful when another research group transfected human $\mathrm{T}$ cells with CXCR4 siRNA using CNTs [59]. In a different strategy, alkylated dendron-functionalized multi-walled (MW) CNTs were used for siRNA delivery [60]. Dendrons with terminal tetraalkylammonium salts imparted a positive charge to MWCNTs, which allowed for efficient siRNA loading combined with the intrinsic cell penetration properties of CNT; the cellular transport of siRNA was remarkably increased. Cationic functionalization also allowed the topical delivery of CNTs. For example, a recent study demonstrated that succinated poly(ethyleneimine) (PEI-SA) functionalized SWCNTs were able to penetrate mouse skin to deliver a siRNA therapeutic to melanoma cells in a C57BL/6 mouse model [61]. Such novel strategies have provided new possibilities for future siRNA delivery and cancer therapy using functionalized CNTs.

\subsubsection{Gold nanoparticles}

Gold nanoparticles are attractive carriers for drug or gene therapeutics, due to their excellent biocompatibility, chemically inert nature, tunable size and shape, large surface area for functionalization, and controllable surface plasmon resonance property. They are usually hybrid systems with a central gold core surrounded by a layer of organic polymer(s) or biomolecules with desirable functionalities for use as nanocarriers. The plasmon resonance 
property of gold nanoparticles offers the possibility of 'on-demand' release of their cargo in response to optical-laser excitation. This property of plasmon resonance has been widely explored in the context of siRNA delivery $[62,63]$. In one study, the nanoparticle was designed to release its cargo nucleic acid by irradiation with near-infrared (NIR) wavelength light $[62,64]$. The surface linker bond between the siRNA gold nanoshell was cleaved by femtosecond pulses of NIR radiation, releasing the siRNA therapeutics [62]. Another approach involved the use of light irradiation in the controlled release of siRNA from surface-coated layer(s) or from covalently attached biomolecules on gold nanoparticle surfaces. The energy absorbed by nanoparticles upon light irradiation increased the temperature on the surface of the gold nanoparticles; this broke the surface layer linkages or the linkage $(\mathrm{Au}-\mathrm{S})$ between nucleic acids and gold, and ultimately released the therapeutic nucleic acid from the nanoparticles $[65,66]$. While both strategies show promise in successful gene delivery, the latter may be more advantageous, since the required power of light (laser) is relatively low and the irradiation time required to release the payload is short.

pH-triggered charge-reversal is a novel technique applied to the controlled release of siRNA from gold nanoparticle-polymer hybrid systems. In this technique, the nanoparticle possesses a negative charge while in the blood stream. The charge reverses to positive following entry into the cell endosome, causing endosomal disruption and siRNA release. Gold nanoparticles are functionalized with specific charge-reversal polymers to render them $\mathrm{pH}$ sensitive. Guo et al. (2010) showed that cy5-siRNA complexed with gold nanoparticles functionalized with charge-reversal polymer poly(allylamine)/ poly(ethyleneimine)/11-mercaptoundecanoic acid (cy5-siRNA/PEI/PAH-Cit/MUA-AuNP) successfully delivered pH-triggered siRNA to HeLa cells. The transfection efficiency of these charge-reversal functional gold nanoparticles was better than commercial transfection agent Lipofectamine 2000, with much lower toxicity to cells [67]. Another strategy utilized glutathione-cleavable disulfide linkages to create siRNA-gold nanoparticles that were sensitive to tumor-relevant glutathione levels [68]. In this study, siRNA was first conjugated to PEG-modified gold nanoparticles via glutathione-cleavable disulfide linkages. Then, nanoparticles were end-modified with poly(aminoester)s (PBAEs) to facilitate efficient nucleic acid delivery. The PBAE-siRNA-AuNPs were able to knockdown the target gene with efficiency comparable to the commercial transfection reagent Lipofectamine 2000.

\subsection{NANOPARTICLE-BASED HUR SIRNA DELIVERY}

The current efforts in our laboratory are focused on developing powerful siRNA delivery systems for the targeted knockdown of HuR (Figure 1). HuR is a nucleocytoplasmic shuttling protein, is overexpressed in several cancers 
including lung cancer, and its overexpression was shown to be a poor prognostic marker in patients diagnosed with lung cancer. Studies from our laboratory demonstrated that HuR knockdown using HuR-siRNA in several lung cancer cell lines significantly reduced cell growth and had a global effect on tumor cell growth inhibition. With enormous interest in the unique characteristics of each of the lipid, polymer, and metal-based nanoparticles, we tried to explore various siRNA delivery systems targeting HuR in cancer cells. This section of the chapter will discuss the characteristics and challenges in the development of HuR siRNA delivery using three different classes of nanocarriers: liposomes, chitosan nanoparticles, and gold nanoclusters.

A
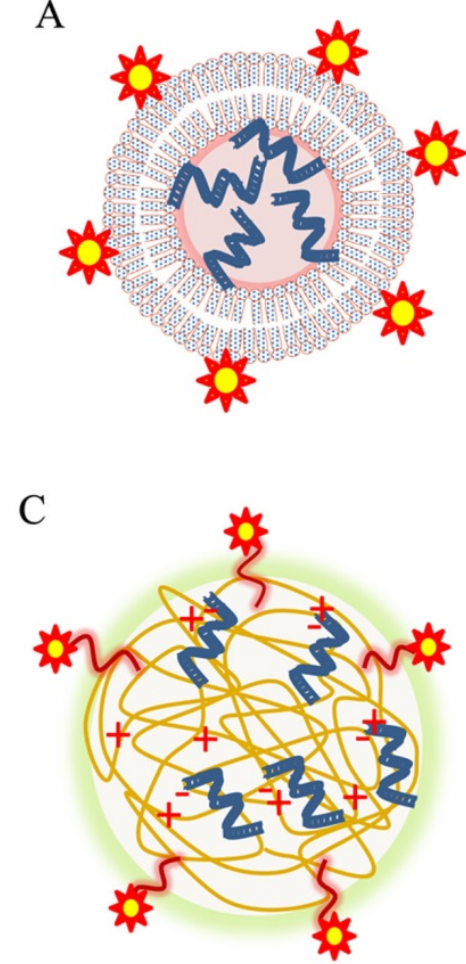

B

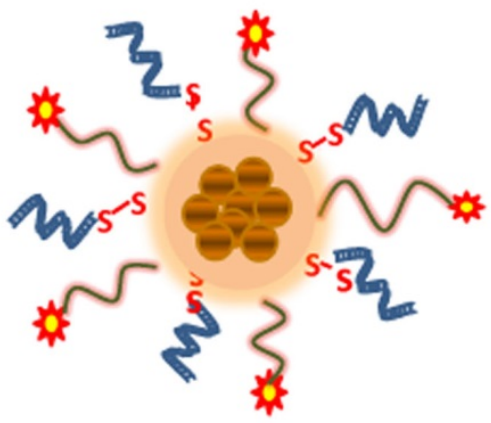

to Targeting Ligand

A siRNA

Linker molecule

Figure 1. HuR siRNA delivery strategies using nanoparticles. (A) Liposome-siRNA complex. siRNA is encapsulated via charge-charge interactions between cationic lipid and negatively charged siRNA, (B) Gold nanocluster-siRNA complex. siRNA is conjugated via glutathione-cleavable disulfide (S-S) bonds, (C) Chitosan nanoparticles encapsulating siRNA. siRNA is entrapped within an ionically crosslinked chitosan-tripolyphosphate nanoparticle. All of the nanoparticle systems are modified with specific ligands for targeted siRNA delivery.

\subsubsection{DOTAP:Chol liposomes}

Since our previous studies with DOTAP:Chol liposomal carriers were successful delivering genes [12-15], we chose this system to deliver siRNA targeting the RNA binding protein $\mathrm{HuR}$ in vitro and in vivo. In vitro studies 
were conducted in many cancer cell lines that overexpress HuR protein. Our results demonstrated successful transfection and knockdown of HuR using siHuR-DOTAP:Chol nanocarriers, followed by an enhanced cell killing effect. Intra-tumoral injection of HuR-siRNA using non-targeted lipid nanoparticles showed promising anti-tumor effects in a mouse xenograft model. We later explored the use of these DOTAP:Chol nanoparticles for the targeted delivery of HuR siRNA, in order to enhance its treatment efficiency. We have introduced folic acid or transferrin molecules as targeting ligands in the DOTAP:Chol liposomes for targeted siRNA delivery. Our first step in the exploration of cationic lipid (DOTAP:Chol) tethered to folate via PEGylated phospholipid linker was tested in folate receptor (FR)-expressing lung cancer cells. We synthesized the FR-targeting cationic liposomes encapsulated with HuR-siRNA (HuR-FNP). After the preparation of HuR-FNP, characterization, FR dependent cellular uptake, cytotoxicity, and gene silencing were investigated to determine the therapeutic efficacy of FNP. Our in vivo pilot studies showed that the FNPs were selectively taken up by the tumor compared with the surrounding organs, when FNPs were administered intravenously into the lung xenograft model.

In the second strategy, TfR-targeted lipid nanoparticles were synthesized in our laboratory. DSPE-PEG-Tf was synthesized by chemical conjugation of thiol-modified transferrin into DSPE-PEG, which is post inserted into DOTAP:Chol lipid nanoparticle to form TfR-NP. The resulting nanoparticle was confirmed for conjugation and subjected to characterization and functional studies. The TEM analysis showed that the TfR-NP were of uniform size, and characterization studies showed sizes of $200-300 \mathrm{~nm}$ and a $+4.0 \mathrm{mV}$ charge [unpublished data]. Our results revealed the efficient and specific delivery of siRNA to the TfR-overexpressing A549 lung cancer cells, with reduced or silent gene expression of HuR at the protein and mRNA levels. This, in turn, showed a global effect on the other HuR-regulated oncoproteins in A549 cells. The specificity of TfR-mediated gene silencing was illustrated using excess human transferrin as a competing agent in the transfection medium. Transferrin blocked the uptake of TfR-NP. Pre-incubation of cells with desferrioxamine enhanced the expression of TfR over the cells which increased the uptake of TfR-NP. We further confirmed these findings by comparing the gene silencing activity in cells expressing different levels of transferrin receptor. TfR-NP exhibited no cytotoxic effect on the normal lung fibroblast line, MRC-9. In vivo studies are currently underway to demonstrate the therapeutic efficacy of the TfR-NP in a lung tumor xenograft model.

\subsubsection{Chitosan nanoparticles}

As an alternative siRNA delivery system, we investigated chitosan nanoparticles for HuR siRNA delivery. Preliminary studies were carried out to prepare chitosan nanoparticles by mixing cationic chitosan polymer with anionic siRNA specific to HuR mRNA to form polyplexes. The complexation 
between chitosan and siRNA was achieved in acidic $\mathrm{pH}$ (5.5) by protonating the free amine groups in chitosan polymer for efficient electrostatic interaction with siRNA. It is important to maintain a proper ratio of siRNA to chitosan (N/P or wt/wt) for efficient complex formation between anionic siRNA and cationic chitosan polymer. We have used medium molecular weight chitosan (Sigma-Aldrich) with a 75-85\% deacetylation degree for the preparation of HuR siRNA nanoparticles. The self-assembled nanoparticles allow for siRNA loading of above $90 \%$ and protect HuR siRNA, as demonstrated by siRNA loading and gel retardation analysis, respectively. The nanocomplex retained the positive charge of chitosan even after siRNA complexation $(+20 \mathrm{mv})$, with particle sizes in the range of $200-300 \mathrm{~nm}$ [unpublished data]. To study the efficiency of siRNA delivery using the chitosan-siHuR complex, we transfected H1299 lung cancer cells that overexpress HuR. At 1:60 wt/wt ratios of siRNA to chitosan, the siHuR-chitosan nanocomplex was able to knockdown HuR with moderate efficiency. Studies have shown that efficiency of transfection with chitosan nanocarrier can be improved if siRNA is encapsulated within ionically crosslinked chitosan nanoparticles [69]. Since the ultimate goal is to improve the transfection efficiency using chitosan nanoparticles targeting $\mathrm{HuR}$, we propose to investigate ionically crosslinked chitosan as siRNA delivery vehicle. HuR siRNA will be incorporated into chitosan-tripolyphosphate (TPP) nanoparticles during the ionic gelation procedure. The anionic counter ion sodium tripolyphosphate will be mixed with the siRNA solution and carefully added to the chitosan solution ( $\mathrm{pH}$ 5.5) under mild stirring. The siRNA to chitosan ratio may need to be re-evaluated for use with chitosan-TPP/siRNA nanoparticles, as the ratio may vary with the change with the introduction of TPP. A fully characterized chitosan-TPP/siRNA system is expected to show enhanced siRNA loading and thereby improve HuR knockdown efficiency. The numerous functional groups available within the chitosan polymer will be utilized to conjugate targeting ligands and/or small molecule therapeutics for cancer treatment. The successful formulation will be used as an alternative, safe, biocompatible and targeted nanocarrier system for HuR siRNA delivery in cancer cells and mouse models, as preliminary steps for their translation.

\subsubsection{Gold nanoparticles}

We are also currently working with HuR-siRNA delivery using gold nanoparticles or nanoclusters. Among different modifications of gold nanoparticles, gold nanoclusters have unique characteristics, such as sub-nanometer size $(<2 \mathrm{~nm}$ in diameter) and prominent photoluminescent properties. Gold nanoclusters modified with polycations or proteins are currently being explored as capping/stabilizing agents for the incorporation of drug molecules or siRNA therapeutics $[70,71]$. In our laboratory, we have synthesized bovine serum albumin-capped gold nanoclusters intended for HuR siRNA delivery in cancer cells. The siRNA can be either electrostatically linked or physically conjugated to the surface coating of the gold nanoclusters. 
Our strategy is to use a thiol-modified HuR siRNA to physically link to the bovine serum albumin (BSA) coating of gold nanoclusters through a glutathione-cleavable disulfide bond. The presence of glutathione in cancer cells allows for disulfide bond breakage and the release of HuR siRNA into the cell cytoplasm. Targeting ligands (transferrin or folic acid) will be conjugated to the surface functional groups of BSA-gold nanoclusters for targeted siRNA delivery. The ultrasmall structure of nanoclusters coupled with the targeting properties will result in enhanced siRNA delivery in cancer cells that overexpress specific receptors. Step-by-step screening of the transfection and gene silencing efficiency of HuR siRNA-gold nanoclusters in cancer cells and tumor models will allow us to develop a novel potential platform for simultaneous siRNA delivery and imaging.

\subsection{CONCLUSIONS}

siRNA, the prominent gene therapy tool for the treatment of cancer, requires safe and efficient delivery vehicles to overcome the barriers to systemic exposure. To date, numerous nanoparticle-based siRNA delivery systems have been developed. Many of these have shown promise in the successful delivery of gene therapeutics intended for cancer therapy. The present chapter discusses the recent progress made in siRNA delivery systems in each major category: liposomes/liposomal variants, polymers, and inorganic nanoparticles. Liposomal systems are highly sought-after nanocarriers for siRNA delivery due their biocompatibility and their clinically proven efficiency of drug delivery. Despite their toxic nature, cationic liposomes are still the best choice, because of their unparalleled capacity for siRNA delivery. Modifications with PEG, the insertion of neutral lipids, and the addition of targeting ligands are cationic liposome modifications that can allow for safe and efficient gene delivery. A recent advancement and liposome variant, the lipid nanoparticle, allows for enhanced protection of siRNA and provides robust gene silencing in tumors. Polymer nanoparticles have been investigated as alternative gene delivery systems because of their versatility and tunable controlled-release. siRNA can be either encapsulated in polymer matrix or electrostatically coupled to cationic polymers. Modification with stimuli-responsive polymers adds additional advantages to polymer nanoparticles for the precise and controlled release of siRNA into the cell cytoplasm. The availability of numerous functional groups in polymers allows for the conjugation of targeting ligands and antibodies for enhanced specificity in siRNA delivery towards cancer cells that overexpress specific receptors. Though toxicity of highly cationic synthetic polymers, such as PEI, restricts their in vivo use, biocompatible polymers, such as chitosan or PLGA, hold promise for safe and efficient nucleic acid delivery. Inorganic nanoparticles have the advantages of multifunctionality and good safety profiles make these nanoparticles attractive as siRNA delivery systems. Along with siRNA delivery, the imaging capabilities 
can be coupled to localize the nanoparticles during the process of siRNA delivery and transfection. Inorganic nanoparticles are smaller in size and can be functionalized to add intracellular cleavable bonds for stimuli-responsive release of siRNA. Our studies demonstrated the advantages of using nanoparticles representing all three categories in delivering HuR siRNA for the treatment of lung and ovarian cancer cells. DOTAP-Chol liposomes demonstrated safe delivery and efficient gene silencing in many types of cancer cells. Ligand-coupled DOTAP:Chol liposomes carrying HuR siRNA enhanced the specificity of gene delivery, producing efficient antitumor effects. Similarly, we expect that our current work investigating chitosan nanoparticles and gold nanoclusters will also strengthen our siRNA therapeutic arsenal for the fight against cancer. Moving forward, we expect major advancements in these gene delivery systems, which will make them strong enough to be tested in clinical settings for cancer gene therapy.

\section{ACKNOWLEDGMENTS}

This study was supported in part by the National Cancer Institute grant R01 CA167516 (RR); by the National Institutes of Health (NIH) grant P20 GM103639-02 (AM) from the COBRE Program of NIH; and by the Jim and Christy Everest Endowed Chair in Cancer Developmental Therapeutics (RR). R.R. is an Oklahoma TSET Research Scholar and holds the Jim and Christy Everest Endowed Chair in Cancer Developmental Therapeutics. 


\section{REFERENCES}

1. T.M. Rana. Nat. Rev. Mol. Cell. Biol. 8 (2007) 23-26.

2. G.R. Devi. Cancer Gene. Ther. 13 (2006) 819-829.

3. R. Kanasty, J.R. Dorkin, A. Vegas, D. Anderson. Nat. Mater. 12 (2013) 967-977.

4. $\quad$ C.E. Thomas, A. Ehrhardt, M.A. Kay. Nat. Rev. Genet. 4 (2003) 346-358.

5. J.-M. Lee, T.-J. Yoon, Y.-S. Cho. Biomed. Res. Int. 2013 (2013) 782041.

6. $\quad$ S. Mallick, J.S. Choi. J. Nanosci. Nanotech. 14 (2014) 755-765.

7. D.A. Balazs, W.T. Godbey. J. Drug Deliv. 2011 (2011) 326497.

8. R.W. Malone, R.L. Felgner, I.M. Verma. Proc. Natl. Acad. Sci. USA 86 (1989) 6077-6081.

9. J. Felgner, M. Martin, Y. Tsai, P.L. Felgner. J. Tiss. Cult. Meth. 15 (1993) 63-68.

10. M. Zhao, H. Yang, X. Jiang, W. Zhou, B. Zhu, Y. Zeng, K. Yao, C. Ren. Mol. Biotechnol. 40 (2008) 19-26.

11. H. Lv, S. Zhang, B. Wang, S. Cui, J. Yan. J. Control. Release 114 (2006) 100-109.

12. R. Ramesh, T. Saeki, N.S. Templeton, L. Ji, L.C. Stephens, I. Ito, D.R. Wilson, Z. Wu, C.D. Branch, J.D. Minna, J.A. Roth. Mol. Ther. 3 (2001) 337-350.

13. I. Ito, L. Ji, F. Tanaka, Y. Saito, B. Gopalan, C.D. Branch, K. Xu, E.N. Atkinson, B.N. Bekele, L.C. Stephens, J.D. Minna, J.A. Roth, R. Ramesh. Cancer Gene Ther. 11 (2004) 733-739.

14. R. Ramesh, I. Ito, Y. Saito, Z. Wu, A.M. Mhashikar, D.R. Wilson, C.D. Branch, J.A. Roth, S. Chada. DNA Cell Biol. 23 (2004) 850-857.

15. S.D. Li, L. Huang. Mol. Pharm. 3 (2006) 579-588.

16. C. Lu, D.J. Stewart, J.J. Lee, L. Ji, R. Ramesh, G. Jayachandran, M.I. Nunez, I.I. Wistuba, J.J. Erasmus, M.E. Hicks, E.A. Grimm, J.M. Reuben, V. Baladandayathupani, N.S. Templeton, J.D. McMannis, J.A. Roth. Plos One 7 (2012) e34833.

17. G. Shim, M.-G. Kim, J.Y. Park, Y.-K. Oh. Asian J. Pharm. Sci. 8 (2013) 72-80.

18. C. Wan, T.M. Allen, P.R. Cullis. Drug Deliv. Transl. Res. 4 (2014) 74-83.

19. Q. Lin, C. Juan, Z. Zhihong, Z. Gang. Nanomedicine (Lond) 9 (2014) 105-120.

20. H.K. Kim, E. Davaa, C.S. Myung, J.S. Park. Int. J. Pharm. 392 (2010) 141-147.

21. S.D. Li, L. Huang. Biochim. Biophys. Acta 1788 (2009) 2259-2266.

22. O. Tirosh, Y. Barenholz, J. Katzhendler, A. Priev. Biophys. J. 74 (1998) 1371-1379.

23. E.A. Ho, M. Osooly, D. Strutt, D. Masin, Y. Yang, H. Yan, M. Bally. J. Pharm. Sci. 102 (2013) 227-236.

24. D. Strumberg, B. Schultheis, U. Traugott. Int. J. Clin. Pharmacol. Ther. 50 (2012) 76-78.

25. https://clinicaltrials.gov/ct2/show/NCT01808638 (Feb 1, 2015).

26. T. Lobovkina, B.J. Gunilla, E. Gonzalez-Gonzalez, P.H. Robyn, L. Devin, L.K. Roger, H.C. Christopher, N.Z. Richard. ACS Nano 5 (2011) 9977-9983.

27. J.B. Lee, K. Zhang, Y.Y. Tam, Y.K. Tam, N.M. Belliveau, V.Y. Sung. Int. J. Cancer 131(2012) E781-E790.

28. K.H. Bae, J.Y. Lee, S.H. Lee, T.G. Park, Y.S. Nam. Adv. Healthc. Mater. 2 (2013) 576-584.

29. G. Grandinetti, A.E. Smith, T.M. Reineke. Mol. Pharm. 9 (2012) 523-538.

30. W. Xia, Y. Li, B. Lou, P.J. Wang, X.L. Gao, C. Lin. J. Nanomaterials 2013 (2013) 384717. 
31. G. Navarro, R.R. Sawant, S. Biswas, S. Essex, C.T. de Ilarduya, V.P. Torchilin. Nanomedicine (Lond) 7 (2012) 65-78.

32. J. Chen, H. Tian, Z. Guo, J. Xia, A. Kano, A. Maruyama, X. Jing, X. Chen. Macromol. Biosci. 9 (2009) 1247-1253.

33. F. Danhier, E. Ansorena, J.M. Silva, R. Coco, A.L. Breton, V. Preat. J. Control. Release 161 (2012) 505-522.

34. Y. Patil, J. Panyam. Int. J. Pharm. 367 (2009) 195-203.

35. W. Hasan, K. Chu, A. Gullapalli, S.S. Dunn, E.M. Enlow, L.C. Luft, S. Tian, M.E. Napier, P.D. Pohlhaus, J.P. Rolland, J.M. DeSimone. Nano. Lett. 12 (2012) 287-292.

36. W.-P. Su, F.-Y. Cheng, D.-B. Shieh, C.-S. Yeh, W.-C. Su. Int. J. Nanomedicine 7 (2012) 4269-4283.

37. J.E. Zuckerman, I. Gritli, A. Tolcher, J.D. Heidel, D. Lim, R. Morgan, B. Chmielowski, A. Ribas, M.E. Davis, Y. Yen. Proc. Natl. Acad. Sci. USA 111 (2014) 11449-11454.

38. J.M. Li, Y.Y. Wang, W. Zhang, H. Su, L.-N. Ji, Z.-W. Mao. Int. J. Nanomedicine 8 (2013) 2101-2117.

39. S. Biswas, V.P. Torchilin. Pharmaceuticals (Basel) 6 (2013) 161-183.

40. J. Wu, W. Huang, Z. He. The Sci. World. J. 2013 (2013) 630-654.

41. L. Albertazzi, L. Gherardini, M. Brondi, S. Sulis, A. Bifone, T. Pizzorusso, G. Matto, G. Bardi. Mol. Pharm. 10 (2013) 249-260.

42. Y. Tang, Y.B. Li, B. Wang, R.Y. Lin, M. van Dongen, D.M. Zurcher, X.Y. Gu, M.M. Banaszak Holl, G. Liu, R. Qi. Mol. Pharm. 9 (2012) 1812-1821.

43. C. Liu, X. Liu, P. Rocchi, F. Qu, J.L. Iovanna, L. Peng. Bioconjugate Chem. 25 (2014) 521-532.

44. H. Katas, H.O. Alpar. J. Control. Release 115 (2006) 216-225.

45. K.-L. Chang, Y. Higuchi, S. Kawakami, F. Yamashita, M. Hashida. Bioconjugate Chem. 21 (2010) 1087-1095.

46. S.K. Tripathi, R. Goyal, P. Kumar, K.C. Gupta. Nanomedicine NBM 8 (2012) 337-345.

47. J. Jung, A. Solaki, K.A. Memoli, K. Kameli, H. Kim, M.A. Drahl, L.J. Williams, H.R. Tseng, K. Lee. Angew. Chem. Int. Ed. Engl. 49 (2010) 103-107.

48. A.M. Derfus, A.A. Chen, D.-H. Min, E. Ruoslahti, S.N. Bhatia. Bioconjugate Chem. 18 (2007) 1391-1396.

49. M.V. Yezhelyev, L.F. Qi, R.M. O'Regan, S. Nie, X.H. Gao. J. Am. Chem. Soc. 130 (2008) 9006-9012.

50. Z. Medarova, M. Kumar, S.W. Ng, A. Moore. Meth. Mol. Biol. 555 (2009) 1-13.

51. C. Boyer, P. Priyanto, P.T. Davis, D. Pissuwan, V. Bulmus, M. Kavallaris, W.Y. Teoh, R. Amal, M. Carroll, R. Woodward, T. St Pierre. J. Mater. Chem. 20 (2010) 255-265.

52. S. Lee, X. Chen. Mol. Imaging 8 (2009) 87-100.

53. H. Meng, M. Liong, T. Xia, Z.X. Li, Z.X. Ji, J.I. Zink, A.E. Nel. ACS Nano 4 (2010) 4539-4550.

54. X. Ma, Y. Zhao, K.W. Ng, Y. Zhao. Chemistry 11 (2013) 15593-155603.

55. S.R. Bhattarai, E. Muthuswamy, A. Wani, M. Brichacek, A.L. Castaneda, S.L. Brock, D. Oupicky. Pharm. Res. 27 (2010) 2556-2568.

56. X. Li, Q.R. Xie, J. Zhang, W. Xia, H. Gu. Biomaterials 32 (2011) 9546-9556.

57. X. Li, Y. Chen, M. Wang, Y. Ma, W. Xia, H. Gu. Biomaterials 34 (2013) 1391-1401. 
58. $\quad$ N.W.S. Kam, Z. Liu, H. Dai. J. Am. Chem. Soc. 127 (2005) 12492-12493.

59. Z. Liu, M. Winters, M. Holodniy, H. Dai. Angew. Chem. Int. Ed. Engl. 46 (2007) 2023-2027.

60. M.A. Herrero, F.M. Toma, K.T. Al-Jamal, K. Kostarelos, A. Bianco, T. Da Ros, F. Bano, L. Casalis, G. Scoles, M. Prato. J. Am. Chem. Soc. 131 (2009) 9843-9848.

61. K.S. Siu, D. Chen, X. Zheng, X. Zhang, N. Johnston, Y. Liu, K. Yuan, J. Koropatnick, E.R. Gillies, W.P. Min. Biomaterials 35 (2014) 3435-3542.

62. G.B. Braun, A. Pallaoro, G. Wu, D. Missilris, J.A. Zasadzinski, M. Tirrell, N.O. Reich. ACS Nano 3 (2009) 2007-2015.

63. R. Huschka, A. Barhoumi, Q. Liu, J.A. Roth, L. Ji, N.J. Halas. ACS Nano 6 (2012) 7681-7691.

64. A. Barhoumi, R. Huschka, R. Bardhan, M.W. Knight, N.J. Halas. Chem. Phys. Lett. 482 (2009) 171-179.

65. L. Poon, W. Zandberg, D. Hsiao, Z. Erno, D. Sen, B.D. Gates, N.R. Branda. ACS Nano 4 (2010) 6395-6403.

66. W. Lu, G. Zhang, R. Zhang, L.G. Flores II, Q. Huang, J.G. Gelovani, C. Li. Cancer Res. 70 (2010) 3177-3188.

67. S. Guo, Y. Huang, Q. Jiang, Y. Sun, L. Deng, Z. Liang, Q. Du, J. Xing, Y. Zhao, P.C. Wang, A. Dong, X.-J. Liang. ACS Nano 4 (2010) 5505-5511.

68. J.-S. Lee, J.J. Green, K.T. Love, J. Sunshine, R. Langer, D.G. Anderson. Nano Lett. 9 (2009) 2402-2406.

69. T.Rojanarata, P. Opanasopit, S. Techaarpornkul, T. Ngawhirunpat, U. Ruktanonchai. Pharm. Res. 25 (2008) 2807-2814.

70. Y. Tao, Z. Li, E. Ju, J. Ren, X. Qu. Nanoscale 5 (2013) 6154-6160.

71. T. Chen, S. Xu, T. Zhao, L. Zhu, D. Wei, Y. Li, H. Zhang, C. Zhao. ACS Appl. Mater. Interfaces 4 (2012) 5766-5774. 
Chapter 7 